International Journal of Pure and Applied Mathematics

Volume 103 No. 3 2015, 511-521

ISSN: 1311-8080 (printed version); ISSN: 1314-3395 (on-line version)

url: http://www.ijpam.eu

doi: http://dx.doi.org/10.12732/ijpam.v103i3.12

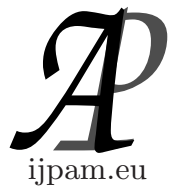

\title{
BLOCK METHOD WITH ONE HYBRID POINT FOR THE SOLUTION OF FIRST ORDER INITIAL VALUE PROBLEMS OF ORDINARY DIFFERENTIAL EQUATIONS
}

\author{
A.U. Fotta ${ }^{1}$, T.J. Alabi ${ }^{2}$, B. Abdulqadir ${ }^{3}$ \\ ${ }^{1}$ Department of Mathematics \\ Adamawa State Polytechnic \\ Yola, Adamawa State, NIGERIA \\ ${ }^{2}$ Department of Mathematics/Statistics \\ Kogi State Polytechnic \\ Lokoja, NIGERIA \\ ${ }^{3}$ Department of Mathematics \\ Federal College of Education \\ Yola, Adamawa State, NIGERIA
}

\begin{abstract}
This paper discussed the development of one - step, one hybrid block method for the solution of first order initial value problems. Two functions were combined to form the basis function which is collocated and interpolated at some selected grid and off-grid points to develop a linear multistep method which is implemented in block form. The paper further investigated the properties of the block method and found it to be convergent. The region of absolute stability was also investigated. The method was tested on some numerical experiments and found to give better approximation than the methods we compared our results with.
\end{abstract}

AMS Subject Classification: 65L05, 65L06, 65D30

Key Words: hybrid, consistent, grid points, off-grid points, convergent, block method, exponential function, interpolation

Received: May 8, 2015

(C) 2015 Academic Publications, Ltd.

$\S_{\text {Correspondence author }}$ url: www.acadpubl.eu 


\section{Introduction}

This paper considered the numerical solution of first order initial value problems of the form

$$
y^{\prime}=f(x, y) ; \quad y\left(x_{0}\right)=y_{0} ; \quad x \in[a, b] .
$$

Here $x_{0}$ is the initial point, $y_{0}$ is the solution at the initial point and $f$ is assumed to be continuous and differentiable within the interval of integration. Moreover it is assumed to satisfy the existence and uniqueness theorem.

Theorem 1.1. (see [1], Existence and Uniqueness Theorem) Let $f(x, y)$ be defined and continuous for all points $(x, y)$ in the region $D$ defined by $a \leq$ $x \leq b,-\infty<y<\infty$. a and $b$ finite, and let there exist a constant $L$ such that, for every $x, y, y^{*}$ such that $(x, y)$ and $\left(x, y^{*}\right)$ are both in $D$.

$$
\left|f(x, y)-f\left(x, y^{*}\right)\right| \leq L\left|y-y^{*}\right| \text {. }
$$

Then, if $y_{0}$ is any given number, there exists a unique solution $y(x)$ of the initial value problem (1), where is $y(x)$ continuous and differentiable for all in D.

Scholars have developed different numerical methods for the solution of initial value problems of ordinary differential equations. Among them are [2, $3,4,5]$ to mention but few. These authors individually implemented their methods such that it gives better error estimation; provide simplified form of coefficient for further analytical work at different points. Lately, scholars adopted method of collocation and interpolation of approximate solution to develop linear multistep method which was implemented in block form. The approximate solution used depend on the nature of the differential equation to be solved, among them are $[6,7,8,9,10,11,12]$. The block method is more efficient than the existing methods such as predictor-corrector method in terms of time of development and execution. Recently, [10, 13, 14, 15, 16] among others, developed hybrid block methods for the solution of initial value problems in ordinary differential equations. The methods gave better stability properties and circumvented the Dahlquist stability condition. Moreover, these methods gave better approximation and they equally reported that lower $\mathrm{k}$ - step method gives better result than higher $\mathrm{k}$ - step method. In this paper, the development of the hybrid block method is carried out using an approximate solution which is a combination of power series and exponential function. It should be noted that our approximate solution considered will be more of exponential function than the one proposed by the authors mentioned above. 


\section{Methodology}

We considered an approximate solution that combines power series and exponential function of the form,

$$
y(x)=\sum_{i=0}^{1} a_{i} e^{i}+\sum_{i=2}^{3} a_{i} e^{-i x}
$$

The first derivative of (2) gives

$$
y^{\prime}(x)=\sum_{i=0}^{1} i a_{i} e^{i-1}-\sum_{i=2}^{3} i a_{i} e^{-i x}
$$

where $a_{i}^{\prime} s$ are unknown constants to be determined. Substituting (3) into (2) gives

$$
f(x, y)=\sum_{i=0}^{1} i a_{i} e^{i-1}-\sum_{i=2}^{3} i a_{i} e^{-i x}
$$

Interpolating (3) at $x_{n+r}, r=0, \frac{1}{2}, 1$ and collocating (4) at points $x_{n+s}, s=$ $0, \frac{1}{2}, 1$ gives a system of nonlinear equation in the form

$$
X A=U
$$

where

$$
\begin{gathered}
X=\left[\begin{array}{cccc}
1 & x_{n} & e^{-2 x_{n}} & e^{-3 x_{n}} \\
0 & 1 & -2 e^{-2 x_{n}} & -3 e^{-3 x_{n}} \\
0 & 1 & -2 e^{-2 x_{n+\frac{1}{2}}} & -3 e^{-3 x_{n+\frac{1}{2}}} \\
0 & 1 & -2 e^{-2 x_{n+1}} & -3 e^{-3 x_{n}+1}
\end{array}\right], \\
A=\left[a_{0}, a_{1}, a_{2}, a_{3}\right]^{T}, U=\left[y_{n}, f_{n}, f_{n+\frac{1}{2}}, f_{n+1}\right]^{T} .
\end{gathered}
$$

Solving (5) for the $a_{i}^{\prime} s$ and substituting back into (4) gives a continuous linear multistep method of the form

$$
\begin{gathered}
y(t)=\alpha_{0} y_{n}+h\left(\beta_{0} f_{n}+\beta_{\frac{1}{2}} f_{n+\frac{1}{2}}+\beta_{1} f_{n+1}\right) \\
t=\frac{x-x_{n}}{h}, f_{n+j}=\left(x_{n}+j h, y\left(x_{n}+j h\right)\right),
\end{gathered}
$$

where:

$$
\alpha_{0}=1
$$




$$
\begin{aligned}
& \beta_{0}=\frac{1}{6} h\left(4 t^{3}-9 t^{2}+6 t\right), \\
& \beta_{\frac{1}{2}}=-\frac{2}{3} h\left(2 t^{3}-3 t^{2}\right), \\
& \beta_{1}=\frac{1}{6} h\left(4 t^{3}-3 t^{2}\right) .
\end{aligned}
$$

Evaluating (6) at $t=0, \frac{1}{2}, 1$ and writing in block form gives a discrete block method of the form

$$
A^{(0)} Y_{M}=E y_{n}+h d f\left(y_{n}\right)+h b F\left(Y_{M}\right)
$$

where

$$
A^{(0)}=\left[\begin{array}{ll}
1 & 0 \\
0 & 1
\end{array}\right], E=\left[\begin{array}{ll}
0 & 1 \\
0 & 1
\end{array}\right], d=\left[\begin{array}{cc}
0 & \frac{5}{24} \\
0 & \frac{1}{6}
\end{array}\right], b=\left[\begin{array}{cc}
\frac{1}{3} & -\frac{1}{24} \\
\frac{2}{3} & \frac{1}{6}
\end{array}\right] .
$$

\section{Analysis of the Basic Properties of the Block Method}

\subsection{Order of the Block Method}

Let the linear operator $L\{y(x): h\}$ associated with the block integrator (7) be defined as

$$
L\{y(x): h\}=A^{(0)} Y_{M}-E y_{n}+h d f\left(y_{n}\right)+h b F\left(Y_{M}\right)
$$

Expanding (8) using Taylor series and comparing the coefficients of $\mathrm{h}$ gives $L\{y(x): h\}=c_{0} y(x)+c_{1} h y^{\prime}(x)+c_{2} h^{2} y^{\prime \prime}(x)+\ldots+c_{p} h^{p} y^{p}(x)+c_{p+1} h^{p+1} y^{p+1}(x)+\ldots$

Definition 1.1. (Order of Block Method) The linear operator L and associated block method are said to be of order $\mathrm{p}$ if $c_{0}=c_{1}=c_{2}=\ldots=c_{p}=$ $0 . c_{p+1} \neq 0 . c_{p+1}$ is called the error constant and implies that the truncation error is given by

$$
t_{n+k}=c_{p+1} h^{p+1} y^{p+1}(x)+0\left(h^{p+2}\right)
$$

for our method

$$
\begin{array}{r}
L\{y(x): h\}=\left[\begin{array}{ll}
1 & 0 \\
0 & 1
\end{array}\right]\left[\begin{array}{l}
y_{n+\frac{1}{2}} \\
y_{n+1}
\end{array}\right]-\left[\begin{array}{ll}
0 & 1 \\
0 & 1
\end{array}\right]\left[y_{n}\right]-h\left[\begin{array}{ccc}
\frac{5}{24} & \frac{1}{3} & -\frac{1}{24} \\
\frac{1}{6} & \frac{2}{3} & \frac{1}{6}
\end{array}\right]\left[\begin{array}{c}
f_{n} \\
f_{n+\frac{1}{2}} \\
f_{n+1}
\end{array}\right] \\
=\left[\begin{array}{l}
0 \\
0
\end{array}\right],
\end{array}
$$


expanding (11) in Taylor series, we find that

$$
\left[\begin{array}{c}
\sum_{j=0}^{\infty} \frac{\left(\frac{1}{2} h\right)^{\mathrm{j}}}{j !} y_{n}^{\prime}-y_{n}-\frac{5 h}{24} y_{n}^{\prime}-\sum_{j=0}^{\infty} \frac{h^{j+1}}{j !} y_{n}^{j+1}\left\{\frac{1}{3}\left(\frac{1}{2}\right)^{j}+\frac{1}{24}(1)^{j}\right\} \\
\sum_{j=0}^{\infty} \frac{(1 h)^{j}}{j !} y_{n}^{\prime}-y_{n}-\frac{1}{6} y_{n}^{\prime}-\sum_{j=0}^{\infty} \frac{h^{j+1}}{j !} y_{n}^{j+1}\left\{\frac{2}{3}\left(\frac{1}{2}\right)^{j}+\frac{1}{6}(1)^{j}\right\}
\end{array}\right]
$$

Hence, the hybrid block method is of order 3 with error constant $=[2.6042(-$ $03),-3.4722(-04)] \mathrm{T}$.

\subsection{Zero-Stability}

Definition 1.2. (Zero-Stability) The block method (7) is said to be zero stable, if the roots $z_{s}, s=1,2, \ldots, k$ of the first characteristics polynomial $\rho(z)$ defined by $\rho(z)=\operatorname{det}\left(z A^{(0)}-E\right)$ satisfies $\left|z_{s}\right| \leq 1$ and if every root with modulus $\left|z_{s}\right|=1$ have multiplicity not exceeding the order of the differential equation. Moreover as, $h \rightarrow 0, \rho(z)=z^{r-\mu}(z-1)^{\mu}$ where $\mu$ is the order of the differential equation, $\mathrm{r}$ is the order of the matrices $A^{(0)}$ and $E$.

$$
\rho(z)=\left|z\left[\begin{array}{ll}
1 & 0 \\
0 & 1
\end{array}\right]-\left[\begin{array}{ll}
0 & 1 \\
0 & 1
\end{array}\right]\right|=\left|\begin{array}{cc}
z & -1 \\
0 & z-1
\end{array}\right|=z(z-1)
$$

Thus, solving for $\mathrm{z}$ in

$$
z(z-1)=0
$$

gives $z_{1}=0, z_{2}=1$. Hence, the block integrator is zero - stable.

\subsection{Consistency}

The block method (7) is consistent since it has order $p=3 \geq 1$.

\subsection{Convergence}

The block method is convergent by the consequence of Dahlquist theorem below.

Theorem 1.2. (see [17]) The necessary and sufficient conditions that a linear multistep method be convergent are that it be consisteznt and zero-stable.

\subsection{Region of Absolute Stability}

Definition 1.3. (Region of Absolute Stability) Region of absolute stability is a region in the complex $z$ plane, where $z=\lambda h$. It is defined as those values 


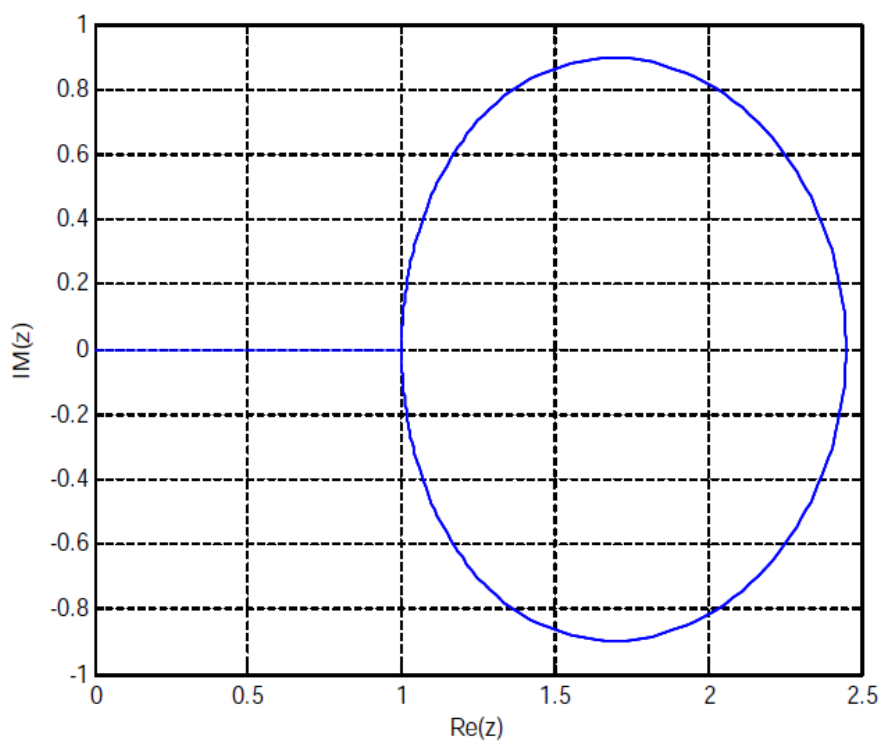

Figure 1: Stability region

of $z$ such that the numerical solution of $y^{\prime}=-\lambda y$ satisfies $j_{j} \rightarrow 0$ as $j \rightarrow \infty$ for any initial condition. To determine the absolute stability region of the new block method, we adopt the boundary locus method. This is achieved by substituting the test equation

$$
y^{\prime}=-\lambda y
$$

into the block formula (7).This gives

$$
A^{(0)} Y_{M}(w)=E y_{n}(w)-h \lambda D y_{n}(w)-h \lambda B Y_{M}(w)
$$

Thus,

$$
\bar{h}=\left(\frac{A^{(0)} Y_{M}(w)-E y_{n}(w)}{D y_{n}(w)-B Y_{M}(w)}\right)
$$

Since $\bar{h}$ is given by $\bar{h}=\lambda h$ and $w=e^{i \theta}$. Equation (16) is our characteristics or stability polynomial for our method, equation (16) is given by

$$
\bar{h}(w)=\left(\frac{1}{12} w^{2}-\frac{1}{12} w\right) h^{2}+\left(-\frac{1}{2} w^{2}-\frac{1}{2} w\right) h+w^{2}
$$

The region of absolute stability is therefore shown in figure 1 below.

Hence the region of absolute stability is region outside the circle. 


\section{Numerical Examples}

The following notations are used in the table.

AENM: Absolute Error in new method

AEA: Absolute Error in [18]

AES: Absolute Error in [10]

AESS: Absolute Error in [11]

\subsection{Problem 1}

We consider a linear first order ordinary differential equation:

$$
y^{\prime}=x-y, \quad y(0)=0, \quad 0 \leq x \leq 1, \quad h=0.1 .
$$

Exact solution: $y(x)=x=e^{-x}-1$. This problem was solved by [18].

Table 1 for Problem 1:

$\begin{array}{ccccc}\text { X } & \text { Exact solution } & \text { Computed solution } & \text { AENM } & \text { AEA } \\ 0.1 & 0.0048374180359596 & 0.0048374180359596 & 3.0358 \mathrm{e}-017 & 0.0000 \\ 0.2 & 0.0187307530779819 & 0.0187307530779819 & 1.3878 \mathrm{e}-017 & 0.0000 \\ 0.3 & 0.0408182206817180 & 0.0408182206817179 & 1.2490 \mathrm{e}-016 & 6.0000 \mathrm{e}-010 \\ 0.4 & 0.0703200460356395 & 0.0703200460356393 & 1.6653 \mathrm{e}-016 & 2.0000 \mathrm{e}-010 \\ 0.5 & 0.1065306597126337 & 0.1065306597126334 & 2.4980 \mathrm{e}-016 & 7.0000 \mathrm{e}-010 \\ 0.6 & 0.1488116360940217 & 0.1488116360940259 & 4.1633 \mathrm{e}-015 & 1.0000 \mathrm{e}-010 \\ 0.7 & 0.1965853037913985 & 0.1965853037914072 & 8.7153 \mathrm{e}-015 & 8.0000 \mathrm{e}-010 \\ 0.8 & 0.2493289641172036 & 0.2493289641172169 & 1.3323 \mathrm{e}-014 & 2.0000 \mathrm{e}-010 \\ 0.9 & 0.3065696597405732 & 0.3065696597405908 & 1.7653 \mathrm{e}-014 & 9.0000 \mathrm{e}-010 \\ 1.0 & 0.3678794411714077 & 0.3678794411714301 & 2.2427 \mathrm{e}-014 & 4.0000 \mathrm{e}-010\end{array}$

\subsection{Problem 2}

We consider a linear first order ordinary differential equation:

$$
y^{\prime}=x y, \quad y(0)=1, \quad 0 \leq x \leq 1, \quad h=0.1 .
$$

Exact solution: $y(x)=e^{\frac{1}{2} x^{2}}$. This problem was solved by [18].

Table 2 for Problem 2: 


$\begin{array}{ccccc}\text { X } & \text { Exact solution } & \text { Computed solution } & \text { AENM } & \text { AEA } \\ 0.1 & 1.0050125208594012 & 1.0050125208594003 & 8.8818 \mathrm{e}-016 & 2.6067 \mathrm{e}-011 \\ 0.2 & 1.0202013400267546 & 1.0202013400267549 & 2.2204 \mathrm{e}-016 & 8.4790 \mathrm{e}-011 \\ 0.3 & 1.0460278599087116 & 1.0460278599087185 & 6.8834 \mathrm{e}-015 & 1.8684 \mathrm{e}-010 \\ 0.4 & 1.0832870676749466 & 1.0832870676749562 & 9.5480 \mathrm{e}-015 & 3.5701 \mathrm{e}-010 \\ 0.5 & 1.1331484530668043 & 1.1331484530668166 & 1.2212 \mathrm{e}-014 & 6.1004 \mathrm{e}-010 \\ 0.6 & 1.1972173631217744 & 1.1972173631217971 & 2.2649 \mathrm{e}-014 & 1.6157 \mathrm{e}-009 \\ 0.7 & 1.2776213132048322 & 1.2776213132048664 & 3.4195 \mathrm{e}-014 & 1.6445 \mathrm{e}-009 \\ 0.8 & 1.3771277643358779 & 1.3771277643359296 & 5.1736 \mathrm{e}-014 & 2.6158 \mathrm{e}-009 \\ 0.9 & 1.4993025000566551 & 1.4993025000567257 & 7.0610 \mathrm{e}-014 & 4.1110 \mathrm{e}-009 \\ 1.0 & 1.6487212706999734 & 1.6487212707000651 & 9.1704 \mathrm{e}-014 & 1.5435 \mathrm{e}-009\end{array}$

\subsection{Problem 3}

Consider the stiff ODE

$$
y^{\prime}=-\lambda y, \quad y(0)=1, \quad \lambda=1, \quad h=0.01 .
$$

Exact solution: $y(x)=e^{-\lambda x}$. This problem was solved by [10].

Table 3 for Problem 3:

$\begin{array}{cc}\mathrm{X} & \text { Exact solution } \\ 0.01 & 0.9900498337491681 \\ 0.02 & 0.9801986733067553 \\ 0.03 & 0.9704455335485082 \\ 0.04 & 0.9607894391523232 \\ 0.05 & 0.9512294245007140 \\ 0.06 & 0.9417645335842486 \\ 0.07 & 0.9323938199059482 \\ 0.08 & 0.9231163463866358 \\ 0.09 & 0.9139311852712282 \\ 0.10 & 0.9048374180359595\end{array}$

Computed solution

0.9900498337491680 0.9801986733067553

0.9704455335485080

0.9607894391523230

0.9512294245007139

0.9417645335842486

0.9323938199059483

0.9231163463866359

0.9139311852712284

0.9048374180359597
AENM

$1.110223 \mathrm{e}-016$

$0.000000 \mathrm{e}+000$

$1.110223 \mathrm{e}-016$

$2.220446 \mathrm{e}-016$

$1.110223 \mathrm{e}-016$

$0.000000 \mathrm{e}+000$

$1.110223 \mathrm{e}-016$

$1.110223 \mathrm{e}-016$

$2.220446 \mathrm{e}-016$

$2.220446 \mathrm{e}-016$
AES

1.842970e-014

$1.088019 \mathrm{e}-014$

$1.110223 \mathrm{e}-016$

$2.220446 \mathrm{e}-014$

$1.798561 \mathrm{e}-014$

$1.076916 \mathrm{e}-014$

$1.798561 \mathrm{e}-014$

$4.440892 \mathrm{e}-014$

$1.743050 \mathrm{e}-014$

$1.054712 \mathrm{e}-014$

\subsection{Problem 4}

Consider the highly stiff ODE

$$
y^{\prime}=-10(y-1)^{2}, \quad y(0)=2 .
$$

Exact solution: $y(x)=1+\frac{1}{(1+10 x)}$. This problem was solved by [11]. 


\begin{tabular}{|c|c|c|c|c|}
\hline $\mathrm{X}$ & $\begin{array}{l}\text { le } 4 \text { for Problem } 4 \text { : } \\
\text { Exact solution }\end{array}$ & Computed solution & AENM & AESS \\
\hline 0.0100 & 1.9090909090909092 & 1.9090906261907747 & $2.829001 \mathrm{e}-007$ & $3.414671 \mathrm{e}-006$ \\
\hline 0.0200 & 1.8333333333333335 & 1.8333329287551785 & $4.045782 \mathrm{e}-007$ & $2.749635 \mathrm{e}-006$ \\
\hline 0.0300 & 1.7692307692307692 & 1.7692303219767005 & $4.472541 \mathrm{e}-007$ & $1.342943 \mathrm{e}-005$ \\
\hline 0.0400 & 1.7142857142857144 & 1.7142852633830512 & $4.509027 \mathrm{e}-007$ & $9.090648 \mathrm{e}-005$ \\
\hline 0.0500 & 1.66666666666666665 & 1.6666662310416001 & $4.356251 \mathrm{e}-007$ & $7.969685 \mathrm{e}-005$ \\
\hline 0.0600 & 1.6250000000000000 & 1.6249995882362918 & $4.117637 \mathrm{e}-007$ & $6.994886 \mathrm{e}-005$ \\
\hline 0.0700 & 1.5882352941176470 & 1.5882349094187826 & $3.846989 \mathrm{e}-007$ & $6.270048 \mathrm{e}-005$ \\
\hline 0.0800 & 1.55555555555555556 & 1.5555551983379770 & $3.572176 \mathrm{e}-007$ & $6.017101 \mathrm{e}-005$ \\
\hline 0.0900 & 1.5263157894736841 & 1.5263154587492180 & $3.307245 \mathrm{e}-007$ & $5.411308 \mathrm{e}-005$ \\
\hline 0.1000 & 1.5000000000000000 & 1.4999996941215274 & $3.058785 \mathrm{e}-007$ & $4.880978 \mathrm{e}-005$ \\
\hline
\end{tabular}

\subsection{Discussion of Results}

This paper considered four numerical examples to test the efficiency of our method. Problem $1 \& 2$ were solved by [18], where they proposed a hybrid method of order six with step-size ratio $\mathrm{r}=1$ and adopted classical RungeKutta method to provide the starting values. Problem $3 \& 4$ were solved by [10] and [11] which are stiff and highly stiff differential equations of order six and five respectively with step size ratio of $\mathrm{r}=1$. The new methods which proposed a block method of order three by combining power series and exponential function as their approximate solution perform better and the error analysis shows that the method was found to be more efficient in terms of accuracy when compared with other methods.

\subsection{Conclusion}

We have developed a new block method with one hybrid points for the solution of first order initial value problems in ordinary differential equations. Our method was found to be zero stable, consistent and convergent. The numerical results show that our method is computationally reliable and gave better accuracy than the existing methods.

\section{References}

[1] P. Henrici. Discrete variable methods in ordinary differential equations. New York: John Wiley and Sons. (1962) 
[2] J. D. Lambert. Computational methods in ordinary differential equations. John y, New York. (1973)

[3] S. O. Fatunla. Numerical methods for initial value problems in ordinary differential Equations. New York Academic Press Inc. (1988).

[4] D.O. Awoyemi, R.A., Ademiluyi, W. Amuseghan. Off - grids exploitation in the development of more accurate method for the solution of ODEs. Journal of Mathematical Physics. 12. (2007). 379 - 386.

[5] Y. Skwame, S. Joshua, E.A. Ibijola. L-stable block hybrid Simpsons methods for Numerical solution of initial value problems in stiff ordinary differential equations. International Journal of Pure and Applied Sciences and Technology. 11(2), (2012).45 - 54.

[6] A. O. Adesanya, M. O. Udoh, A.M. Alkali. A new block predictor - corrector Algorithm for the solution of : American Journal of Computational Mathematics, 2, (2012). 341- 344. DOI:10.4236/ajcm.2012.24047

[7] M.R.Odekunle, A.O. Adesanya, J. Sunday. A new block integrator for the solution of Initial value problems of first-order ODEs. International Journal of Pure and Applied Science Technology. 11(1), (2012). 92 - 100.

[8] A.O. Adesanya, M.O. Udoh, A.M. Ajileye. A new hybrid block method for the Solution of general third order initial value problems of ordinary differential equations. International Journal of Pure and Applied Mathematics. 86(2), (2013).365 - 375. DOI: http://dx.doi.org/10.12732/ijpam.v86i2.11

[9] A.A. James, A.O. Adesanya, S. Joshua. Continuous block method for the Solution of second Order initial value problems of ordinary differential equations. International Journal of Pure and Applied Mathematics. 83, (2013).405 - 416, DOI: http://dx.doi.org/10.12732/ijpam.v83i3.3

[10] J. Sunday, M.R. Odekunle, A.O. Adesanya, A.A. James. Extended block Integrator for first Order stiff and oscillatory differential equations.American Journal of Computational and Applied Mathematics, 3(6), (2013).283 - 290. DOI: 10.5923/j.ajcam.20130306.03

[11] J. Sunday, A.O. Adesanya, M.R. Odekunle. A self-starting four step fifth order Block Integrator for stiff and oscillatory differential equations. Journal of Mathematics and Computer Science. 4(1),(2014).73 - 84. 
[12] M.K. Fasasi, A.O. Adesanya, S.O. Adee. Block numerical integrator for the Solution of International Journal of Pure and Applied Mathematics. 92,(2014). 87 - 97. DOI:http://dx.doi.org/10.12732/ijpam.v92i1.7

[13] J. Sunday, M.R Odekunle, A.O. Adesanya. Order six block integrator for the Solution of first Order ODEs. International Journal of Mathematical Science and Computing. 3(1)1, (2013). 87 - 96.

[14] R.K. Adebayo, A.E. Umar. Generalized rational approximation method via Pade Approximants for the solutions of IVPs with singular solutions and stiff differential Equations. Journal of Mathematical Sciences. 2(1). (2013). 327 - 368 .

[15] S.A. Okunuga, A.B. Sofoluwe, J.O. Ehigie. Some block numerical schemes for Solving initial Value problems in ODEs. Journal of Mathematical Sciences. 2(1).(2013). 387 - 402.

[16] A.A. Momoh, A.O. Adesanya, K.M. Fasasi, A. Tahir. A new numerical integrator for the Solution of stiff first order ordinary differential equations, Engineering Mathematics letter, 5. (2014)

[17] G.G. Dahlquist. Convergence and stability in the numerical integration of ordinary differential equations. Math. Scand. 4. (1956). 33-50.

[18] E.A. Areo, R.A. Ademiluyi, P.O. Babatola. Sixth-Order Hybrid Block Method for the numerical solution of first order initial value problems. Mathematical Theory and Modeling. 3(8), 2013, 113 - 120. 
